\title{
Turbulence Locality and Granularlike Fluid Shear Viscosity in Collisional Suspensions
}

\author{
Diego Berzi ${ }^{1}$ and Luigi Fraccarollo ${ }^{2}$ \\ ${ }^{1}$ Politecnico di Milano, 20133 Milano, Italy \\ ${ }^{2}$ Università di Trento, 38123 Trento, Italy \\ (Received 26 June 2015; published 3 November 2015)
}

\begin{abstract}
We reanalyze previous experimental measurements of solid volume fraction, mean velocity, and velocity fluctuations in collisional suspensions of plastic cylinders and water flowing over inclined, erodible beds. We show that the particle pressure scales with the granular temperature, as predicted by kinetic theory of granular gases. The assumption that the particle shear stress is also well predicted by kinetic theory permits us to determine the fluid shear stress and the effective fluid viscosity from the experiments. The fluid viscosity can be decomposed into turbulent and granularlike components: the turbulent viscosity can be modeled using a mixing length, which is a decreasing function of the local volume fraction and does not depend upon the distance from the bed; the granularlike viscosity, associated with the transfer of momentum due to the conjugate motion of the fluid mass added to the particles, can be modeled by replacing the particle density with the density of the added fluid mass in the viscosity of kinetic theory.
\end{abstract}

DOI: 10.1103/PhysRevLett.115.194501

PACS numbers: 47.55.Kf, 47.57.E-, 47.57.Gc, 83.80.Fg

Modeling the transport of many solid particles in a turbulent shearing flow is crucial to a large number of civil and industrial applications. Two-phase continuous mathematical models are feasible [1], but they require, among other things, the determination of closures for both the particle and the fluid stresses. In this Letter, we restrict our analysis to collisional suspensions, in which the particleimmersed weight is supported by collisions and the particle inertia is dominant [2-4].

There is a general belief that the turbulence of the interstitial fluid is suppressed, at least at large solid volume fractions, when the particle inertia is important [4,5]. However, a quantification of this effect is still lacking and turbulence models in suspensions largely rely on expressions valid in absence of particles. For instance, in mixing length approaches applied to mixtures flowing over rigid or erodible beds, the origin of turbulence is often taken to be near the bed itself-i.e., nonlocal turbulence $[2,6,7]$.

Kinetic theory of granular gases [8-10] provides constitutive relations for the particle stresses, which have been tested against discrete numerical simulations in the absence of interstitial fluid up to large solid volume fractions, near the jamming transition [11-13]. Tests of kinetic theory against physical experiments, however, are scarce, and even more so when the presence of the interstitial fluid cannot be neglected. This has to do with the inherent difficulties in obtaining reliable measurements of solid volume fraction and velocity and distinguishing between the mean component and fluctuations, with the latter playing a key role in the expressions for the stresses of kinetic theory.

Recently, detailed experimental measurements of solid volume fraction, particle mean velocity, and particle velocity fluctuations have been performed [14] on steady, fully developed, inclined, collisional flows of monosized plastic cylinders (mass density $\rho_{p}=1510 \mathrm{~kg} / \mathrm{m}^{3}$, diameter of the equivalent sphere $d=3.35 \mathrm{~mm}$, and heightto-diameter ratio 0.8 ) and water (mass density $\rho_{f}=$ $1000 \mathrm{~kg} / \mathrm{m}^{3}$ and molecular viscosity $\eta_{f}=10^{-3} \mathrm{~Pa} \mathrm{~s}$ ) over erodible beds at an angle $\phi$ with respect to the horizontal [Fig. 1(a)]. Examples of the measured profiles along the direction $y$ perpendicular to the bed of solid volume fraction $\nu$, mean velocity $u$ in the direction $x$ parallel to the bed, and square root of the granular temperature $T$ one third of the mean square of the particle velocity fluctuations - are reported in Fig. 1(b).

The particle momentum balance in the direction perpendicular to the bed reads $[2,4]$

$$
p^{\prime}=-\left(\rho_{p}-\rho_{f}\right) \nu g \cos \phi
$$

where $p$ is the particle pressure, $g$ is the gravitation acceleration, and the prime indicates a derivative with respect to $y$. Here, we have assumed for simplicity that the particle normal stresses are isotropic [15]. We can then obtain the distribution of the particle pressure from a numerical integration of the profile of solid volume fraction [Fig. 1(c)]. With this and the measured profile of granular temperature, we can test the equation of state of kinetic theory [9],

$$
\frac{p}{\rho_{p} T}=4 \nu^{2} g_{0}\left(\frac{1}{4 \nu g_{0}}+\frac{1+e}{2}\right),
$$

where $g_{0}$ is the radial distribution function at contact and $e$ is the effective coefficient of restitution [16], which takes 


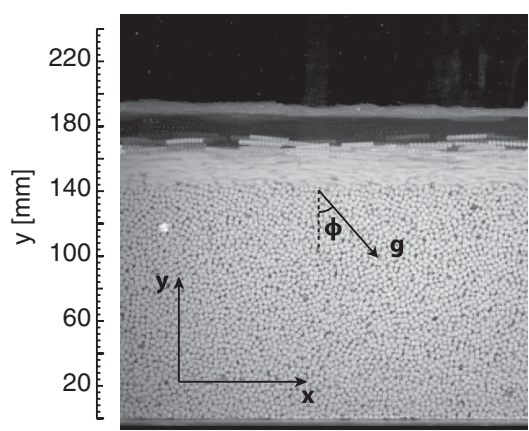

(a)

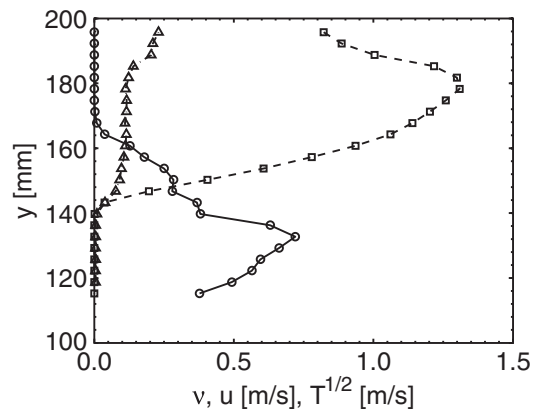

(b)

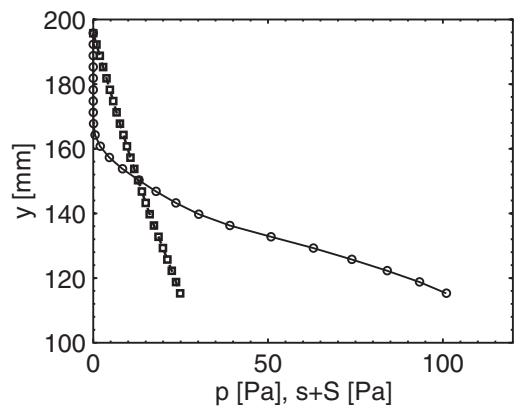

(c)

FIG. 1. (a) Typical side view of the experiments with the frame of reference. (b) Profiles of solid volume fraction (circles), mean $x$ velocity (squares), and square root of the granular temperature (triangles) in one experiment. Because of poor statistics, the measurements of solid volume fraction near the bed are not reliable. In the present Letter, the analysis is therefore limited to the region between the free surface and the first location at which the measured solid volume fraction exceeds the value at random close packing for spheres ( $y$ is approximately between 140 and $200 \mathrm{~mm}$ in this example). (c) Profiles of particle pressure (circles) and total shear stress of the mixture (squares) numerically obtained by integrating the profiles of Fig. 1(b).

into account the role of friction in collisions. Here, for simplicity, we employ the radial distribution function at contact suggested by Torquato [17], which is singular at the random close packing for spheres $(\nu=0.64)$. We take $e$ to be 0.6 , as appropriate for plastic spheres having a normal coefficient of restitution of about 0.9 and interparticle friction of about 0.1 [18]. Using different singularities in the radial distribution function at contact [12] or different coefficients of restitution has very little effect on the equation of state of Eq. (2), at least for $\nu$ less than 0.5 . Figure 2 shows the comparisons between the measured equation of state and Eq. (2). Here, and in what follows, the measurements have been obtained from 77 runs characterized by different combinations of angles of inclination, particle flow rates, and fluid flow rates, and with no evidence of flow instabilities. The collapse of the data suggests that the particle pressure scales with the granular temperature, so kinetic theory provides the correct framework to describe the particle stresses in the presence of an interstitial fluid also. The agreement of Eq. (2) with the measurements is good, considering that that expression is

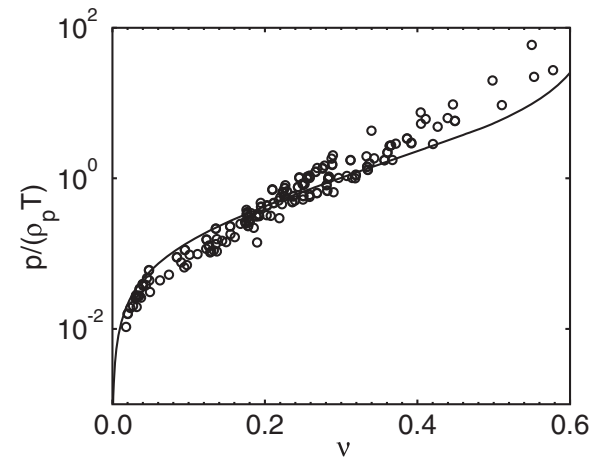

FIG. 2. Measured (circles) equation of state for the particles. The solid line represents Eq. (2). in principle valid for spheres, not cylinders. Equation (2) underpredicts the measurements at solid volume fractions larger than 0.5 , where, however, the measurements are scarce and less reliable.

We can also obtain the distribution of the total shear stress of the mixture [Fig. 1(c)], i.e., the sum of the particle shear stress $s$ and the liquid shear stress $S$, by numerically integrating the mixture momentum balance in the flow direction $[2,4]$,

$$
(s+S)^{\prime}=-\left[\rho_{p} \nu+\rho_{f}(1-\nu)\right] g \sin \phi .
$$

Given that the equation of state of kinetic theory agrees fairly well with the measurements, we assume that the particle shear stress can also be predicted by kinetic theory [9] as

$$
s=\rho_{p} \frac{8 J \nu^{2} g_{0}}{5 \pi^{1 / 2}} d T^{1 / 2} u^{\prime}
$$

where $J$ is a known function of the coefficient of restitution and the solid volume fraction [19]. The prefactor to the shear rate $u^{\prime}$ in Eq. (4) is the granular viscosity, i.e., the transport coefficient associated with the transfer of momentum due to the particle velocity fluctuations. From the experimental measurements, we can then evaluate the particle shear stress and subtract it from the total shear stress of the mixture to obtain the fluid shear stress $S$; with this, we evaluate the effective fluid viscosity $\eta=S / u^{\prime}$, where we have assumed that the derivative with respect to $y$ of the mean $x$ velocity of the fluid is equal to that of the particles. Previous numerical solutions of particle-liquid mixtures shows that this is a reasonable assumption $[2,7,20]$.

We now assume that the effective fluid viscosity is the sum of two contributions [21]: (i) a turbulent hydrodynamic contribution $\eta_{\text {turb}}$, which in unidirectional shear flows is often modeled using a mixing length approach $[2,7,23,24]$ as 


$$
\eta_{\text {turb }}=\rho_{f}(1-\nu) l_{m}^{2} u^{\prime}
$$

where $l_{m}$ is the mixing length; (ii) a granularlike contribution $\eta_{\text {gran }}$, which is due to the fact that, when particles fluctuate, a certain mass of fluid is stuck with them and is subject to conjugate motion-i.e., the added mass effect [25-27]. When particles collide, pressure impulses are generated in the interstitial fluid, causing fluid accelerations; this process very much resembles the exchange of particle momentum in collisions. We therefore assume that this granularlike viscosity has the same expression of the granular viscosity of Eq. (4), with the particle mass density substituted by the density of the added fluid mass associated with the motion of the particles. For the latter, we adopt the analytical expression obtained using the potential flow approximation [27,28], so that

$$
\eta_{\text {gran }}=\rho_{f} \frac{1+2 \nu}{2(1-\nu)} \frac{8 J \nu^{2} g_{0}}{5 \pi^{1 / 2}} d T^{1 / 2}
$$

When scaled using the product of the fluid density, the particle diameter, and the square root of the granular temperature, the measured effective fluid viscosity agrees with Eq. (6) for solid volume fractions greater than 0.3 (Fig. 3), where the granularlike viscosity dominates. The deviation from Eq. (6) at solid volume fractions less than 0.3 indicates that there the turbulent viscosity cannot be neglected.

An expression for the mixing length in the turbulent contribution to the effective fluid viscosity can be obtained from Eq. (5) using the measured solid volume fraction and shear rate, with $\eta_{\text {turb }}=\eta-\eta_{\text {gran }}$ and the granularlike viscosity evaluated from Eq. (6). The obtained mixing length is always less than one particle diameter, and decreases with the increasing solid volume fraction (Fig. 4). Best fitting with the experimental points gives

$$
\frac{l_{m}}{d}=3(0.64-\nu)^{3}
$$

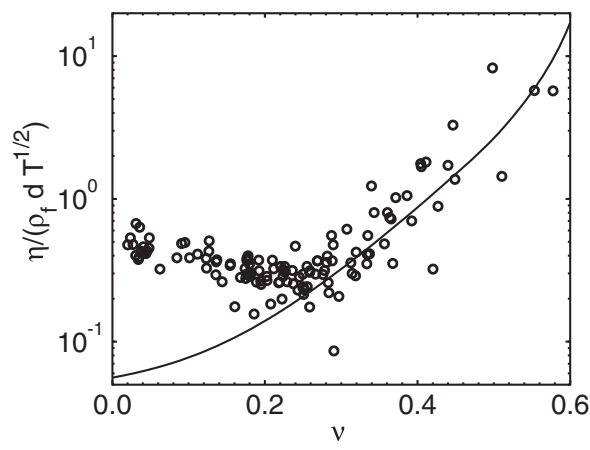

FIG. 3. Measured (circles) effective fluid viscosity scaled with the square root of the granular temperature. The solid line is the scaled granularlike viscosity of Eq. (6).
This result is consistent with the idea that, in collisional suspensions, turbulence originates locally near the surface of the particles, and the presence of the particles provides an upper limit to the size of the turbulent eddies of the order of the mean interparticle distance. It is worth mentioning that the mean interparticle distance is also 2 orders of magnitude larger than the Kolmogorov scale. When the strength of the shearing fluid increases, we expect a transition to a turbulent suspension and also a corresponding development of large-scale turbulence in presence of the particles, as in the experiments of Revil-Baudard et al. [24].

There are two limiting cases that apply to the steady and fully developed flow considered here, if we neglect the conduction of energy associated with the particle velocity fluctuations, which is important only in regions a few diameters thick that are close to rigid boundaries [29]. In the granular limit, the production of fluctuation energy associated with the work of the particle shear stress balances exactly the energy dissipated in collisions due to the particle inelasticity. With the constitutive relations of kinetic theory [9], this implies a simple algebraic relation between the granular temperature and the square of the shear rate [30],

$$
\frac{T}{d^{2} u^{\prime 2}}=\frac{2 J}{15\left(1-e^{2}\right)}
$$

In the turbulent limit, the energy production associated with the influence of the turbulent eddies on the particle fluctuations balances the energy dissipation associated with the drag exerted on the particles by the fluid [20]. This gives $T \propto S /\left[\rho_{f}(1-\nu)\right][31,32]$, where we have taken $S /\left[\rho_{f}(1-\nu)\right]$ to be a measure of the intensity of the fluid turbulent fluctuations. Introducing the fluid effective viscosity, and taking $\eta \approx \eta_{\text {turb }}$, gives, with Eq. (5),

$$
\frac{T}{d^{2} u^{\prime 2}}=3.5\left(\frac{l_{m}}{d}\right)^{2}
$$

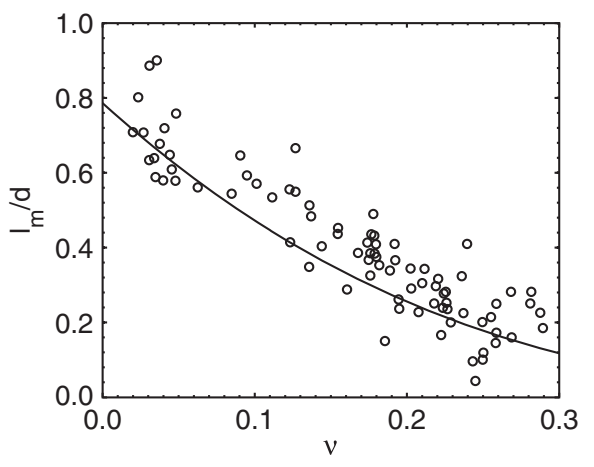

FIG. 4. Dimensionless mixing length as obtained from the experimental measurements (circles). The solid line corresponds to Eq. (7). 


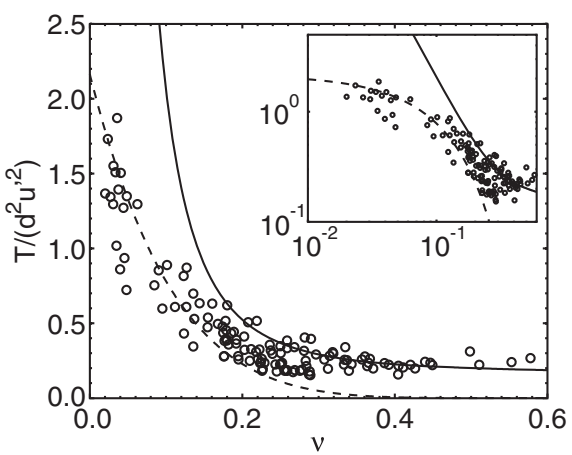

FIG. 5. Measured (circles) scaled granular temperature and theoretical predictions in the granular [Eq. (8), solid line] and turbulent [Eq. (9), dashed line] limit. Inset: same data in a log-log scale.

where the numerical factor 3.5 permits a good fitting with the data. Equation (9), lumped in with Eq. (5), provides a turbulent viscosity proportional to fluid density, the square root of granular temperature, and particle diameter, confirming the analysis of Chen and Louge [33] for numerical simulations [31]. The numerical value of the fluid turbulent shear stress [31] would give a mixing length of about $0.1 d$ at $\nu=0.3$, in excellent agreement with Fig. 4 .

The experimental measurements agree with Eq. (8) at solid volume fractions larger than 0.3 and with Eq. (9) at solid volume fractions less than 0.2 (Fig. 5). In between, the above-mentioned four mechanisms responsible for producing and dissipating the particle fluctuation energy are equally important.

Expressing the effective fluid viscosity as the sum of the turbulent and the granularlike contributions of Eqs. (5) and (6) gives

$$
\frac{\eta}{\rho_{f} d^{2} u^{\prime}}=(1-\nu)\left(\frac{l_{m}}{d}\right)^{2}+\frac{1+2 \nu}{2(1-\nu)} \frac{8 J \nu^{2} g_{0}}{5 \pi^{1 / 2}}\left(\frac{T}{d^{2} u^{\prime 2}}\right)^{1 / 2} .
$$

Figure 6 shows the comparison between the data obtained from the measurements and Eq. (10), when the scaled granular temperature is given by either Eq. (8) or Eq. (9). While the turbulent contribution to the effective fluid viscosity decreases with the solid volume fraction, the granularlike contribution increases: this results in a minimum of the effective fluid viscosity at a solid volume fraction of about 0.3 . The presence of a minimum in the effective viscosity was also observed in the case of turbulent-collisional suspensions by Revil-Baudard et al. [24].

In this Letter, we have suggested that the effective fluid viscosity in collisional suspensions has two components: one associated with the turbulence generated near the surface of the particles and one associated with the transfer of momentum of the fluid mass in conjugate motion with

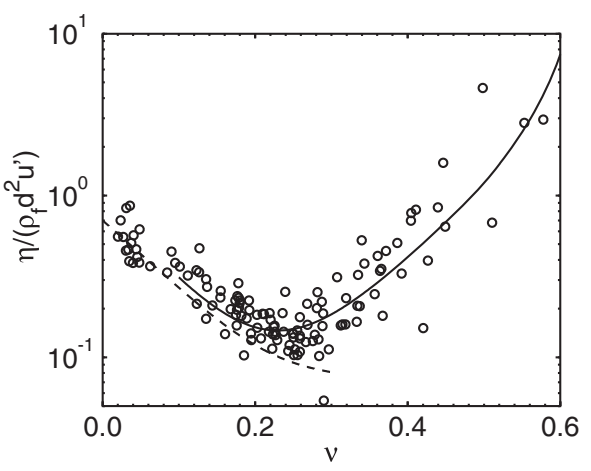

FIG. 6. Measured (circles) and theoretical (lines) effective fluid viscosity scaled with the shear rate. The theoretical predictions are those of Eq. (10) when the scaled granular temperature is given by Eq. (8) (solid line) and Eq. (9) (dashed line).

the fluctuating particles. We have modeled the first contribution using a mixing length approach, and shown that the mixing length is local, as it does not scale with distances from boundaries. The mixing length is less than one diameter and decreases with increase in the solid volume fraction. We have introduced a granularlike viscosity to model the second contribution to the effective fluid viscosity, by replacing the particle mass density with the density of the added mass of the fluid in the expression of the granular viscosity of the particles of kinetic theory. Finally, we have shown how the granular temperature scales in the turbulent and granular limits of the effective fluid viscosity.

[1] C. Crowe, T. Troutt, and J. Chung, Annu. Rev. Fluid Mech. 28, 11 (1996).

[2] J. Jenkins and D. Hanes, J. Fluid Mech. 370, 29 (1998).

[3] D. Berzi, J. Hydraul. Eng. 137, 1200 (2011).

[4] D. Berzi and L. Fraccarollo, Phys. Fluids 25, 106601 (2013).

[5] R. Bagnold, Proc. R. Soc. A 225, 49 (1954).

[6] O. Duran, B. Andreotti, and P. Claudin, Phys. Fluids 24, 103306 (2012).

[7] F. Chiodi, P. Claudin, and B. Andreotti, J. Fluid Mech. 755, 561 (2014).

[8] J. Jenkins and S. Savage, J. Fluid Mech. 130, 187 (1983).

[9] V. Garzó and J. W. Dufty, Phys. Rev. E 59, 5895 (1999).

[10] J. T. Jenkins, Granular Matter 10, 47 (2007).

[11] D. Vescovi, D. Berzi, P. Richard, and N. Brodu, Phys. Fluids 26, 053305 (2014).

[12] D. Berzi and D. Vescovi, Phys. Fluids 27, 013302 (2015).

[13] D. Berzi and J. Jenkins, Soft Matter 11, 4799 (2015).

[14] H. Capart and L. Fraccarollo, Geophys. Res. Lett. 38, L20402 (2011).

[15] In the experiments, the ratio of the mean square of the particle velocity fluctuations along $x$ and $y$, and therefore the ratio of the associated normal stresses, is between 2 and 4 for solid volume fractions less than 0.3 , and approaches 
one (isotropy) at larger solid volume fractions. Therefore, the isotropic component of the normal stresses is between 1.5 and 2.5 times the normal stress perpendicular to the bed when $\nu<0.3$. The agreement of the experiments with the theoretical curve in Fig. 2 would improve if we took this into account.

[16] J. Jenkins and C. Zhang, Phys. Fluids 14, 1228 (2002).

[17] S. Torquato, Phys. Rev. E 51, 3170 (1995).

[18] S. Foerster, M. Louge, H. Chang, and K. Allia, Phys. Fluids 6, 1108 (1994).

[19] $J=(1+e) / 2+(\pi / 32)\left[5+2(3 e-1)(1+e) \nu g_{0}\right][5+4(1+$ $\left.e) \nu g_{0}\right] /\left\{[24-(1-e)(11-e)] \nu^{2} g_{0}^{2}\right\}$, from Garzó and Dufty [9].

[20] T.-J. Hsu, J. Jenkins, and P.-F. Liu, Proc. R. Soc. A 460, 2223 (2004).

[21] The viscous contribution reported by Boyer et al. [22] is negligible in the collisional suspensions examined here.

[22] F. Boyer, E. Guazzelli, and O. Pouliquen, Phys. Rev. Lett. 107, 188301 (2011).
[23] M. Muste, K. Yu, I. Fujita, and R. Ettema, Water Resour. Res. 41, W10402 (2005).

[24] T. Revil-Baudard, J. Chauchat, D. Hurther, and P.-A. Barraud, J. Fluid Mech. 767, 1 (2015).

[25] R. Zenit, M. Hunt, and C. Brennen, J. Fluid Mech. 353, 261 (1997).

[26] R. Zenit, M. Hunt, and C. Brennen, Appl. Sci. Res. 58, 305 (1997).

[27] A. Armanini, H. Capart, L. Fraccarollo, and M. Larcher, J. Fluid Mech. 532, 269 (2005).

[28] H. Lamb, Hydrodynamics, 6th ed. (Cambridge University Press, Cambridge, UK, 1932).

[29] V. Kumaran, J. Fluid Mech. 599, 121 (2008).

[30] J. Jenkins and D. Berzi, Granular Matter 12, 151 (2010).

[31] R. Verberg and D. Koch, Phys. Fluids 18, 083303 (2006).

[32] H. Xu, R. Verberg, D. Koch, and M. Louge, J. Fluid Mech. 618, 181 (2009).

[33] X. Chen and M. Louge, Int. J. Heat Mass Transfer 51, 5108 (2008). 